\title{
A bounded-error approach to simultaneous state and actuator fault estimation for a class of nonlinear systems
}

\author{
Mariusz Buciakowski ${ }^{\mathrm{a}, *}$, Marcin Witczak ${ }^{\mathrm{a}}$, Vicenç Puig ${ }^{\mathrm{b}}$, Damiano Rotondo ${ }^{\mathrm{c}}$, \\ Fatiha Nejjari ${ }^{\mathrm{b}}$, Józef Korbicz ${ }^{\mathrm{a}}$ \\ ${ }^{a}$ Institute of Control and Computation Engineering, University of Zielona Góra, \\ ul. Podgórna 50, 65-246 Zielona Góra, Poland \\ ${ }^{b}$ Automatic Control Department, Universidad Politécnica de Cataluña, Rambla Sant \\ Nebridi, 10, 08222 Terrassa, Spain \\ ${ }^{c}$ Center for Autonomous Marine Operations and Systems (NTNU-AMOS), \\ Department of Engineering Cybernetics, Norwegian University of Science and Technology, \\ Trondheim, Norway
}

\begin{abstract}
This paper proposes an approach for the joint state and fault estimation for a class of uncertain nonlinear systems with simultaneous unknown input and actuator faults. This is achieved by designing an unknown input observer combined with a set-membership estimation in the presence of disturbances and measurement noise. The observer is designed using quadratic boundedness approach that is used to overbound the estimation error. Sufficient conditions for the existence and stability of the proposed state and actuator fault estimator are expressed in the form of linear matrix inequalities (LMIs). Simulation results for a quadruple-tank system show the effectiveness of the proposed approach.

Keywords: Fault detection, fault isolation, quadratic boundedness, nonlinear systems, bounded-error estimation, observer
\end{abstract}

\section{Introduction}

Fault detection and isolation (FDI) procedures have become a very attractive topic in the last decades due to the increasing demand for safety and reliability

\footnotetext{
* Corresponding author

Email address: M.Buciakowski@issi.uz.zgora.pl (Mariusz Buciakowski)
} 
in control engineering systems. However, the presence of nonlinearities in control systems still constitutes a major challenge to model-based FDI. In recent years, some results on FDI for nonlinear systems have been obtained. Some of these works are based on nonlinear observers [1, 2, 3, while others are based on parity space approaches [4. The majority of these FDI approaches have been derived only for some classes of nonlinear systems such as state affine [5], input affine [6] or bilinear systems [7], among others. Apart from analyticalmodel-based solutions there is a solid line of research devoted to developing data-driven models for FDI. This can be realised, e.g., with linear parametric models [8], neural networks [9], fuzzy logic [10] or recursive kernel learning approach [11]. The last approach deserves special attention because, as reported 15 in [1], it outperforms neural networks and its computational framework has several appealing properties. Moreover, its efficiency was proven in the soft sensors design [12], which can be directly applied for FDI purposes.

Another important issue is the fact that in real applications, it is not easy to directly apply the existing FDI schemes because of the presence of uncertainties, disturbances, and noise [13. In addition, it is not always possible to get information about disturbances and noises acting on the system. A solution to such cases can be obtained by assuming bounded uncertainties and using set-membership (or bounded-error) methods [14. In these methods, noise, disturbances and uncertainties acting on the model parameters are assumed to be unknown but bounded with a priori known bound. Using this bounded description, the set of state/parameter values that are consistent with the measurements and the model structure can be calculated. Then, whenever a measurement is not consistent with any of the members of this set, a fault is detected. The bounded-error approximations are achieved using different geometrical regions such as ellipsoids [15], parallelotopes [16], polytopes [17, orthotopes [18], zonotopes [19] or a combination of them. More recently, set-membership fault detection techniques have been investigated by several authors 20, 14, 21, 22, 23.

It should be also underlined that the use of on-line fault estimation is es- 
sential for all active fault compensation approaches. A number of suitable fault estimation methods, essentially observer-based [24, 25, 26, 27, Kalman filterbased [28, or parameter identification-based [29] are used. In [30, a fault estimation scheme for nonlinear systems that can be modeled in linear parameter varying form is presented. In [20, an observer scheme that simultaneously 40 estimate the state and the fault is considered. In [31, a robust fault estimation approach for nonlinear discrete-time systems using unknown input observer is presented.

A class of nonlinear systems of special attention is the so-called Lipschitz ones [32, 33], in which the mathematical model of the system satisfies the Lip45 schitz continuity condition. Many observer-based FDI approaches have been reported for this class of nonlinear systems, such as unknown input observers [34], adaptive observers [25], descriptor system approaches [35] and high-gain observers [36]. In [3] and [25], a fault diagnosis for Lipschitz nonlinear systems by using adaptive observer has been presented. In [37], a sliding mode observer has been designed for nonlinear Lipschitz bounded systems and recently, nonlinear observers for one-sided Lipschitz systems have been considered, see, e.g, $[38,, 39,40]$.

A very interesting approach for simultaneous state and unknown input estimation was proposed in [41]. In particular, the authors considered a Lipschitz system with a nonlinearity split into known and unknown parts. It is also worth noting that the system output equation has a direct input feed-through. The design observer design procedure boils down to transforming the original system into an equivalent form without a direct feed-through, decoupling the effect of unknown nonlinearity and providing asymptotic estimates of the system input

${ }_{60}$ and state. A similar approach was proposed in [42] for nonlinear time-delay systems. It is important to underline that the above work considers unknown but bounded delays, which constitute the main source of uncertainty, while the nonlinearity is treated as a disturbance, which is suitably decoupled. A further extension of the above general framework was recently proposed in [32]]. ${ }_{65}$ Contrarily to [41] , the usual Lipschitz condition is replaced by one-sided and 
quadratically inner-bounded ones, which extends its applicability to a wider class of nonlinear systems. A similar strategy was employed for reduced-order observer in [33]. Taking into account the fact that the estimated unknown input can be perceived as unknown faults, the above approach can be adapted for simultaneous state and fault estimation.

The contribution of this paper is to propose a novel approach for simultaneous state and actuator fault estimation, which can be perceived as a fusion of [42, [41] and the above-mentioned set-membership strategies. In the proposed strategy, a set-membership approach is used to provide a feasible set of states and actuator faults that are consistent with the measurements supposing that the external system disturbances are unknown but bounded. This means that the main contribution of this work is the design of an unknown input observer combined with a set-membership estimation in the presence of disturbances and measurement noises. It is worth mentioning that the proposed technique for state and fault reconstruction is different than the one presented in [31] where the objective was to determine an optimal state and fault estimation in the sense of $\mathcal{H}_{\infty}$ norm. In this work, the observer is designed using a quadratic boundedness (QB) approach that is used to overbound the estimation error. Sufficient conditions for the existence and stability of the proposed state and actuator fault estimator are expressed in the form of linear matrix inequalities (LMIs), which can be solved using available computational packages [43].

The paper is organised as follows: Section 2 presents task set-up regarding the problem being considered. In Section 3 , the joint state and fault estimation approach is proposed. Section 4 provides the computational framework used for obtaining a feasible state and fault set that is consistent with the current measurements. Section 5 presents the final procedure for the boundederror estimation. In Section 6, the application of the proposed approach to a quadruple-tank system is presented. Finally, Section 7 concludes the paper.

Notation: The following notation will be used throughout this paper.

- $k$-sample index; 
- $t$ - time;

- $\mathbb{R}^{n}, \mathbb{R}^{r}, \mathbb{R}^{m}, \mathbb{R}^{q}-n, r, m, q$ dimensional real vector space;

- $\boldsymbol{x}_{k} \in \mathbb{R}^{n}, \hat{\boldsymbol{x}}_{k} \in \mathbb{R}^{n}$ - system state vector and its estimate;

- $\boldsymbol{z}_{k} \in \mathbb{R}^{n}$ - observer state vector;

- $\boldsymbol{u}_{k} \in \mathbb{R}^{r}$ - input vector;

- $\boldsymbol{y}_{k} \in \mathbb{R}^{m}$ - output vector;

- $\boldsymbol{d}_{k} \in \mathbb{R}^{q}$ - unknown input disturbance vector;

- $\boldsymbol{f}_{k} \in \mathbb{R}^{r}, \hat{\boldsymbol{f}}_{k} \in \mathbb{R}^{r}$ - actuator fault and its estimate;

- $\boldsymbol{g}\left(\boldsymbol{x}_{k}, \boldsymbol{u}_{k}\right)$ - continuous, differentiable nonlinear function with respect to $\boldsymbol{x}_{k}$ and $\boldsymbol{u}_{k}$

- $\boldsymbol{w}_{k} \in \mathbb{R}^{n}$ - exogenous disturbance vector;

- $\boldsymbol{e}_{k} \in \mathbb{R}^{n}, \boldsymbol{e}_{f, k} \in \mathbb{R}^{r}$ - state and fault estimation error respectively;

- $\boldsymbol{P} \succ \mathbf{0}(\boldsymbol{P} \prec \mathbf{0})$ - positive definite (negative definite) square matrix;

- $\alpha>0(\alpha<0)-$ positive (negative) scalar;

- $\eta$ - Lipschitz constant;

- $\rho$ - one-side Lipschitz constant;

- $\boldsymbol{I}$ - identity matrix;

- $\boldsymbol{A}^{T}$ - transpose of matrix $\boldsymbol{A}$;

- $\boldsymbol{A}^{-1}$ - inverse of matrix $\boldsymbol{A}$;

- $\boldsymbol{\nu} \in \mathcal{E}_{\boldsymbol{\nu}}, \mathcal{E}_{\boldsymbol{\nu}}=\left\{\boldsymbol{\nu}: \boldsymbol{\nu}^{T} \boldsymbol{Q}_{\boldsymbol{\nu}} \boldsymbol{\nu} \leq 1\right\}, \boldsymbol{Q}_{\boldsymbol{\nu}} \succ 0$ - ellipsoidal set; 


\section{Problem set-up}

The system under consideration is described by a nonlinear discrete-time state-space form

$$
\begin{aligned}
\boldsymbol{x}_{k+1} & =\boldsymbol{A} \boldsymbol{x}_{k}+\boldsymbol{B} \boldsymbol{u}_{k}+\boldsymbol{D} \boldsymbol{d}_{k}+\boldsymbol{g}\left(\boldsymbol{x}_{k}, \boldsymbol{u}_{k}\right)+\boldsymbol{B} \boldsymbol{f}_{k}+\boldsymbol{W}_{1} \boldsymbol{w}_{k}, \\
\boldsymbol{y}_{k} & =\boldsymbol{C} \boldsymbol{x}_{k}+\boldsymbol{W}_{2} \boldsymbol{w}_{k},
\end{aligned}
$$

where $\boldsymbol{A}, \boldsymbol{B}, \boldsymbol{D}, \boldsymbol{W}_{1}, \boldsymbol{W}_{2}, \boldsymbol{C}$ are constant matrices of appropriate dimensions. Note that the disturbance input vector $\boldsymbol{w}_{k}$, includes system disturbances, measurements noises, etc. The function $\boldsymbol{g}\left(\boldsymbol{x}_{k}, \boldsymbol{u}_{k}\right)$ is nonlinear and is assumed to be differentiable with respect to $\boldsymbol{x}_{k}$ and $\boldsymbol{u}_{k}$. Using the differential mean value theorem [44], it can be shown that

$$
\boldsymbol{g}(\boldsymbol{a}, \boldsymbol{u})-\boldsymbol{g}(\boldsymbol{b}, \boldsymbol{u})=\boldsymbol{M}_{x, u}(\boldsymbol{a}-\boldsymbol{b}),
$$

with

$$
\boldsymbol{M}_{x, u}=\left[\begin{array}{c}
\frac{\partial g_{1}}{\partial x}\left(\boldsymbol{c}_{\boldsymbol{1}}, \boldsymbol{u}\right) \\
\vdots \\
\frac{\partial g_{n}}{\partial x}\left(\boldsymbol{c}_{\boldsymbol{n}}, \boldsymbol{u}\right)
\end{array}\right],
$$

where $\boldsymbol{c}_{1}, \ldots, \boldsymbol{c}_{n} \in \mathrm{Co}(\boldsymbol{a}, \boldsymbol{b}), \boldsymbol{c}_{i} \neq \boldsymbol{a}, \boldsymbol{c}_{i} \neq \boldsymbol{b}, i=1, \ldots, n$. Based on the fact that $\boldsymbol{x}_{k}$ is bounded, i.e., $\boldsymbol{x}_{k} \in \mathbb{X} \subset \mathbb{R}^{n}$, it is possible to show that

$$
\bar{a}_{i, j} \geq \frac{\partial g_{i}(\boldsymbol{x})}{\partial x_{j}} \geq \underline{a}_{i, j}, \quad i=1, \ldots, n, \quad j=1, \ldots, n,
$$

where $\bar{a}_{i, j} \geq \underline{a}_{i, j}$ are known bounds.

Let us define the following set of matrices

$$
\mathbb{M}=\left\{\boldsymbol{M} \in \mathbb{R}^{n \times n} \mid \bar{a}_{i, j} \geq m_{i, j} \geq \underline{a}_{i, j}, i, j=1, \ldots, n,\right\}
$$

that is useful in order to define the following assumption(s):

Assumption 1: There exists a matrix $\boldsymbol{M} \in \mathbb{M}$ such that:

$$
(\boldsymbol{g}(\boldsymbol{a}, \boldsymbol{u})-\boldsymbol{g}(\boldsymbol{b}, \boldsymbol{u}))^{T}(\boldsymbol{a}-\boldsymbol{b}) \leq(\boldsymbol{a}-\boldsymbol{b})^{T} \boldsymbol{M}^{T}(\boldsymbol{a}-\boldsymbol{b})
$$


Assumption 2: There exists a matrix $M \in \mathbb{M}$ such that:

$$
(\boldsymbol{g}(\boldsymbol{a}, \boldsymbol{u})-\boldsymbol{g}(\boldsymbol{b}, \boldsymbol{u}))^{T}(\boldsymbol{g}(\boldsymbol{a}, \boldsymbol{u})-\boldsymbol{g}(\boldsymbol{b}, \boldsymbol{u})) \leq(\boldsymbol{a}-\boldsymbol{b})^{T} \boldsymbol{M}^{T} \boldsymbol{M}(\boldsymbol{a}-\boldsymbol{b})
$$

It is worth noting that, if $\boldsymbol{M}^{T} \boldsymbol{M}=\eta^{2} \boldsymbol{I}$ then the relation underlying Assumption 2 (cf. [45, 46] ) becomes a usual Lipschitz condition [37, 47, 48, 49] with $\eta$ being

120 a Lipschitz constant. This appealing property makes the employed strategy more general than the ones presented in the literature [37, 47, 48, 49]. Moreover, a significant progress was recently obtained in the observer design for nonlinear systems by introducing the so-called one-sided Lipschitz condition [50, which means that a wider spectrum of systems can be tackled with the new approach.

125 Indeed, if $\boldsymbol{M}=\rho \boldsymbol{I}$, then the relation underlying Assumption 1 becomes a one-sided Lipschitz condition, which is imposed along with the usual Lipschitz condition (see [50] for further details and explanations). Thus, it is evident that this appealing property makes again the employed strategy more general than those presented in the literature (see [50] and the references therein). Moreover, for further analysis, three additional assumptions are considered for the system:

\section{Assumption 3:}

$$
\begin{aligned}
& \varepsilon_{k}=\boldsymbol{f}_{k+1}-\boldsymbol{f}_{k}, \\
& \varepsilon_{k} \in \mathcal{E}_{\boldsymbol{\varepsilon}}, \mathcal{E}_{\boldsymbol{\varepsilon}}=\left\{\boldsymbol{\varepsilon}: \boldsymbol{\varepsilon}^{T} \boldsymbol{Q}_{\boldsymbol{\varepsilon}} \boldsymbol{\varepsilon} \leq 1\right\}, \boldsymbol{Q}_{\boldsymbol{\varepsilon}} \succ 0 .
\end{aligned}
$$

Assumption 4:

$$
\operatorname{rank}(\boldsymbol{D})=\operatorname{rank}(\boldsymbol{C D})=q, \quad q \leq m
$$

\section{Assumption 5:}

$$
\boldsymbol{w}_{k} \in \mathcal{E}_{\boldsymbol{w}}, \mathcal{E}_{\boldsymbol{w}}=\left\{\boldsymbol{w}: \boldsymbol{w}^{T} \boldsymbol{Q}_{\boldsymbol{w}} \boldsymbol{w} \leq 1\right\}, \boldsymbol{Q}_{\boldsymbol{w}} \succ 0
$$

Assumption 3 is required to attain a suitable fault estimation quality. The value of $\varepsilon_{k}$ is unknown but bounded in an ellipsoidal set 31. Assumption 4 is used to decouple the effect of an unknown input (see, e.g., [51, 52, for further details). Finally, Assumption 5 states that the external disturbances are unknown but 
associated with the so-called bounded-error or set-membership approaches [14, 53. Contrarily to 31, where the objective was to obtain an optimal state and fault estimator in the sense of $\mathcal{H}_{\infty}$ norm, the idea is to obtain an estimator that will provide a feasible set of states and faults that are consistent with the measurements. This means that the proposed scheme gives knowledge about upper and lower bounds of the system states and faults that can be perceived as worst-case situations. This information is especially important from the viewpoint of fault-tolerant control [54, 55].

Given the above preliminaries and assumptions, the objective of the subsequent part of this paper is to recall the state and fault estimation strategy for the class of nonlinear discrete-time systems (1)-2 that was recently proposed by the authors in [31]. The approach proposed in the current paper will use a similar estimation structure but its derivation and estimation objectives are different. Furthermore, the proposed scheme will be extended to cope with the

bounded-error estimation, i.e., instead of a single estimate, a set of estimates that are consistent with current measurements is provided.

The general estimator structure proposed in 31] is as follows

$$
\begin{aligned}
\boldsymbol{z}_{k+1} & =\boldsymbol{N} \boldsymbol{z}_{k}+\boldsymbol{G} \boldsymbol{u}_{k}+\boldsymbol{L} \boldsymbol{y}_{k}+\boldsymbol{T} \boldsymbol{B} \hat{\boldsymbol{f}}_{k}+\boldsymbol{T} \boldsymbol{g}\left(\hat{\boldsymbol{x}}_{k}, \boldsymbol{u}_{k}\right) \\
\hat{\boldsymbol{x}}_{k} & =\boldsymbol{z}_{k}-\boldsymbol{E} \boldsymbol{y}_{k} \\
\hat{\boldsymbol{f}}_{k+1} & =\hat{\boldsymbol{f}}_{k}+\boldsymbol{F}\left(\boldsymbol{y}_{k}-\boldsymbol{C} \hat{\boldsymbol{x}}_{k}\right)
\end{aligned}
$$

where $\boldsymbol{T}=\boldsymbol{I}+\boldsymbol{E} \boldsymbol{C}$. Thus, under Assumption 4, it can be shown that for:

$$
\boldsymbol{E}=-\boldsymbol{D}\left[(\boldsymbol{C D})^{T}(\boldsymbol{C D})\right]^{-1}(\boldsymbol{C D})^{T}
$$

and $\boldsymbol{G}=\boldsymbol{T} \boldsymbol{B}$, the following relations can be formulated [31]:

$$
\begin{aligned}
& T D=0, \\
& K=N E+L, \\
& N=T A-K C .
\end{aligned}
$$


By defining the state and fault estimation errors as $\boldsymbol{e}_{k}=\boldsymbol{x}_{k}-\hat{\boldsymbol{x}}_{k}$ and $\boldsymbol{e}_{f, k}=\boldsymbol{f}_{k}-$

$\hat{\boldsymbol{f}}_{k}$, the following augmented vectors can be introduced: $\overline{\boldsymbol{e}}_{k+1}=\left[\boldsymbol{e}_{k+1}^{T}, \boldsymbol{e}_{f, k+1}^{T}\right]^{T}$ and $\boldsymbol{v}_{k}=\left[\boldsymbol{w}_{k}^{T}, \boldsymbol{\varepsilon}_{k}^{T}, \boldsymbol{w}_{k+1}^{T}\right]^{T}$. As a result, the joint state and fault estimation error obeys 31:

$$
\overline{\boldsymbol{e}}_{k+1}=\boldsymbol{X} \overline{\boldsymbol{e}}_{k}+\boldsymbol{Y} \boldsymbol{s}_{k}+\boldsymbol{Z} \boldsymbol{v}_{k}
$$

with $\boldsymbol{s}_{k}=\boldsymbol{g}\left(\boldsymbol{x}_{k}, \boldsymbol{u}_{k}\right)-\boldsymbol{g}\left(\hat{\boldsymbol{x}}_{k}, \boldsymbol{u}_{k}\right)$ where

$$
\begin{gathered}
X=\bar{A}-\bar{K} \bar{C}=\left[\begin{array}{cc}
T A & T B \\
0 & I
\end{array}\right]-\left[\begin{array}{l}
\boldsymbol{K} \\
F
\end{array}\right]\left[\begin{array}{ll}
C & 0
\end{array}\right], \quad Y=\left[\begin{array}{l}
T \\
0
\end{array}\right], \\
Z=\bar{W}-\bar{K} \bar{V}=\left[\begin{array}{ccc}
T W_{1} & 0 & E W_{1} \\
0 & I & 0
\end{array}\right]-\left[\begin{array}{c}
K \\
F
\end{array}\right]\left[\begin{array}{lll}
W_{2} & 0 & 0
\end{array}\right] .
\end{gathered}
$$

Given the general structure of the estimator along with its estimation error (16), a novel design procedure will be presented in the subsequent section.

\section{Novel observer design}

The main objective of this section is to show the design procedure of an observer for the system (1)-(2) using the scheme proposed in the previous section. To settle this problem, QB is employed [56, 57, 58. The main idea behind using this paradigm is the fact that the approach proposed in this paper has to provide a feasible state and fault set that is consistent with the current measurements. As demonstrated in [56], QB can be used efficiently for outerbounding the state estimation error. Thus, a suitable extension has to be developed in order to with the problem defined above.

From (7) and (9) it can be observed, that $\boldsymbol{v}_{k} \in \mathcal{E}_{\boldsymbol{v}}$, where

$$
\begin{aligned}
& \mathcal{E}_{\boldsymbol{v}}=\left\{\boldsymbol{v}: \boldsymbol{v}^{T} \boldsymbol{Q}_{\boldsymbol{v}} \boldsymbol{v} \leq 1\right\}, \\
& \boldsymbol{Q}_{\boldsymbol{v}}=\frac{1}{3} \operatorname{diag}\left(\boldsymbol{Q}_{\boldsymbol{w}}, \boldsymbol{Q}_{\boldsymbol{\varepsilon}}, \boldsymbol{Q}_{\boldsymbol{w}}\right) .
\end{aligned}
$$

For the purpose of further analysis, let $\boldsymbol{P} \succ \mathbf{0}$, which makes it possible to formulate the following definitions (cf. [56]): 
Definition 1. The system 16 is strictly quadratically bounded with $\boldsymbol{P} \succ \mathbf{0}$ for all allowable $\boldsymbol{v}_{k} \in \mathcal{E}_{\boldsymbol{v}}, k \geq 0$, if $\overline{\boldsymbol{e}}_{k}^{T} \boldsymbol{P} \overline{\boldsymbol{e}}_{k}>1$ implies $\overline{\boldsymbol{e}}_{k+1}^{T} \boldsymbol{P} \overline{\boldsymbol{e}}_{k+1}<\overline{\boldsymbol{e}}_{k}^{T} \boldsymbol{P} \overline{\boldsymbol{e}}_{k}$ for any $\boldsymbol{v}_{k} \in \mathcal{E}_{\boldsymbol{v}}$.

This means that strict quadratic boundedness ensures that the Lyapunov function $V_{k}=\overline{\boldsymbol{e}}_{k}^{T} \boldsymbol{P} \overline{\boldsymbol{e}}_{k}$ decreases, i.e., $V_{k+1}<V_{k}$, for 16] and any $\boldsymbol{v}_{k} \in \mathcal{E}_{\boldsymbol{v}}$ when $V_{k}>1$ (cf. [56]).

Before providing the main result of this section, let us remind the following lemma [59]:

Lemma 1. The following statements are equivalent

1. There exist $\boldsymbol{X} \succ \mathbf{0}$ and $\boldsymbol{W} \succ \mathbf{0}$ such that

$$
\boldsymbol{V}^{T} \boldsymbol{X} \boldsymbol{V}-\boldsymbol{W} \prec \mathbf{0},
$$

2. There exist $\boldsymbol{X} \succ \mathbf{0}, \boldsymbol{W} \succ \mathbf{0}$ and $\boldsymbol{U}$ such that

$$
\left[\begin{array}{cc}
-\boldsymbol{W} & \boldsymbol{V}^{T} \boldsymbol{U}^{T} \\
\boldsymbol{U} \boldsymbol{V} & \boldsymbol{X}-\boldsymbol{U}-\boldsymbol{U}^{T}
\end{array}\right] \prec \mathbf{0} .
$$

175 Theorem 1. The following statements are equivalent:

1. The system (16) is strictly quadratically bounded with $\boldsymbol{P} \succ \mathbf{0}$ for all allowable $\boldsymbol{v}_{k} \in \mathcal{E}_{\boldsymbol{v}}$;

2. There exists $\boldsymbol{H}, \boldsymbol{U}, \boldsymbol{P} \succ \mathbf{0}, 0<\gamma<1, \alpha>0, \beta>0$ such that for all $M \in \mathbb{M}$ the following condition is satisfied:

$\left[\begin{array}{ccccc}\boldsymbol{S}_{1} & -\alpha \boldsymbol{V}^{T} & \mathbf{0} & \overline{\boldsymbol{A}}^{T} \boldsymbol{P}-\overline{\boldsymbol{C}}^{T} \boldsymbol{H}^{T} & \boldsymbol{V}^{T} \boldsymbol{M}^{T} \boldsymbol{U}^{T} \\ -\alpha \boldsymbol{V} & -\beta \boldsymbol{I} & \mathbf{0} & \boldsymbol{Y}^{T} \boldsymbol{P} & \mathbf{0} \\ \mathbf{0} & \mathbf{0} & -\gamma \boldsymbol{Q}_{\boldsymbol{v}} & \overline{\boldsymbol{W}}^{T} \boldsymbol{P}-\overline{\boldsymbol{V}}^{T} \boldsymbol{H}^{T} & \mathbf{0} \\ \boldsymbol{P} \overline{\boldsymbol{A}}-\boldsymbol{H} \overline{\boldsymbol{C}} & \boldsymbol{P} \boldsymbol{Y} & \boldsymbol{P} \overline{\boldsymbol{W}}-\boldsymbol{H} \overline{\boldsymbol{V}} & -\boldsymbol{P} & \mathbf{0} \\ \boldsymbol{U} \boldsymbol{M} \boldsymbol{V} & \mathbf{0} & \mathbf{0} & \mathbf{0} & \beta \boldsymbol{I}-\boldsymbol{U}-\boldsymbol{U}^{T}\end{array}\right] \prec \mathbf{0}$

where $\boldsymbol{S}_{1}=-\boldsymbol{P}+\gamma \boldsymbol{P}+\alpha \boldsymbol{V}^{T}\left(\boldsymbol{M}+\boldsymbol{M}^{T}\right) \boldsymbol{V}$ with $\boldsymbol{H}=\boldsymbol{P} \overline{\boldsymbol{K}}$. 
Proof. Using Definition 1 and the fact that $\boldsymbol{v}_{k}^{T} \boldsymbol{Q}_{\boldsymbol{v}} \boldsymbol{v}_{k} \leq 1$ (cf. (19)), it is possible to write:

$$
\boldsymbol{v}_{k}^{T} \boldsymbol{Q}_{\boldsymbol{v}} \boldsymbol{v}_{k}<\overline{\boldsymbol{e}}_{k}^{T} \boldsymbol{P} \overline{\boldsymbol{e}}_{k}, \Rightarrow \overline{\boldsymbol{e}}_{k+1}^{T} \boldsymbol{P} \overline{\boldsymbol{e}}_{k+1}-\overline{\boldsymbol{e}}_{k}^{T} \boldsymbol{P} \overline{\boldsymbol{e}}_{k}<0
$$

As a consequence, using (16) and defining $\overline{\boldsymbol{v}}_{k}=\left[\overline{\boldsymbol{e}}_{k}^{T}, \boldsymbol{s}_{k}^{T}, \boldsymbol{v}_{k}^{T}\right]^{T}$, it can be shown that

$$
\overline{\boldsymbol{v}}_{k}^{T}\left[\begin{array}{ccc}
\boldsymbol{X}^{T} \boldsymbol{P} \boldsymbol{X}-\boldsymbol{P} & \boldsymbol{X}^{T} \boldsymbol{P} \boldsymbol{Y} & \boldsymbol{X}^{T} \boldsymbol{P} \boldsymbol{Z} \\
\boldsymbol{Y}^{T} \boldsymbol{P} \boldsymbol{X} & \boldsymbol{Y}^{T} \boldsymbol{P} \boldsymbol{Y} & \boldsymbol{Y}^{T} \boldsymbol{P} \boldsymbol{Z} \\
\boldsymbol{Z}^{T} \boldsymbol{P} \boldsymbol{X} & \boldsymbol{Z}^{T} \boldsymbol{P} \boldsymbol{Y} & \boldsymbol{Z}^{T} \boldsymbol{P} \boldsymbol{Z}
\end{array}\right] \overline{\boldsymbol{v}}_{k}<\mathbf{0} .
$$

Following Assumption 1, it is evident that

$$
\boldsymbol{s}_{k}^{T} \boldsymbol{e}_{k} \leq \boldsymbol{e}_{k}^{T} \boldsymbol{M} \boldsymbol{e}_{k}=\frac{1}{2} \boldsymbol{e}_{k}^{T}\left(\boldsymbol{M}+\boldsymbol{M}^{T}\right) \boldsymbol{e}_{k} .
$$

Bearing in mind that

$$
\boldsymbol{e}_{k}=\boldsymbol{V} \overline{\boldsymbol{e}}_{k}=\left[\begin{array}{ll}
\boldsymbol{I} & \mathbf{0}
\end{array}\right]\left[\begin{array}{c}
\boldsymbol{e}_{k} \\
\boldsymbol{e}_{f, k}
\end{array}\right],
$$

inequality 25 can be written as

$$
\boldsymbol{s}_{k}^{T} \boldsymbol{V} \overline{\boldsymbol{e}}_{k} \leq \frac{1}{2} \overline{\boldsymbol{e}}_{k}^{T} \boldsymbol{V}^{T}\left(\boldsymbol{M}+\boldsymbol{M}^{T}\right) \boldsymbol{V} \overline{\boldsymbol{e}}_{k},
$$

which, for any $\alpha>0$, is equivalent to

$$
\alpha \overline{\boldsymbol{v}}_{k}^{T}\left[\begin{array}{crr}
\boldsymbol{V}^{T}\left(\boldsymbol{M}+\boldsymbol{M}^{T}\right) \boldsymbol{V} & -\boldsymbol{V}^{T} & \mathbf{0} \\
-\boldsymbol{V} & \mathbf{0} & \mathbf{0} \\
\mathbf{0} & \mathbf{0} & \mathbf{0}
\end{array}\right] \overline{\boldsymbol{v}}_{k} \geq \mathbf{0} .
$$

Similarly, from Assumption 2 it can be shown that

$$
\boldsymbol{s}_{k}^{T} \boldsymbol{s}_{k} \leq \boldsymbol{e}_{k}^{T} \boldsymbol{M}^{T} \boldsymbol{M} \boldsymbol{e}_{k},
$$

which, for any $\beta>0$, can be written as

$$
\beta \overline{\boldsymbol{v}}_{k}^{T}\left[\begin{array}{ccc}
\boldsymbol{V}^{T} \boldsymbol{M}^{T} \boldsymbol{M} \boldsymbol{V} & \mathbf{0} & \mathbf{0} \\
\mathbf{0} & -\boldsymbol{I} & \mathbf{0} \\
\mathbf{0} & \mathbf{0} & \mathbf{0}
\end{array}\right] \overline{\boldsymbol{v}}_{k} \geq \mathbf{0} .
$$


From (23), and in particular from $\boldsymbol{v}_{k}^{T} \boldsymbol{Q}_{\boldsymbol{v}} \boldsymbol{v}_{k}<\overline{\boldsymbol{e}}_{k}^{T} \boldsymbol{P} \overline{\boldsymbol{e}}_{k}$, it is evident that for $\gamma>0$

$$
\gamma \overline{\boldsymbol{v}}_{k}^{T}\left[\begin{array}{ccc}
-\boldsymbol{P} & \mathbf{0} & \mathbf{0} \\
\mathbf{0} & \mathbf{0} & \mathbf{0} \\
\mathbf{0} & \mathbf{0} & \boldsymbol{Q}_{\boldsymbol{v}}
\end{array}\right] \overline{\boldsymbol{v}}_{k}<\mathbf{0}
$$

Now, using the S-procedure [60] for (24), (27), 29) and (30), it can be concluded that

$$
\overline{\boldsymbol{v}}_{k}^{T}\left[\begin{array}{ccc}
\boldsymbol{S}_{2} & \boldsymbol{X}^{T} \boldsymbol{P} \boldsymbol{Y}-\alpha \boldsymbol{V}^{T} & \boldsymbol{X}^{T} \boldsymbol{P} \boldsymbol{Z} \\
\boldsymbol{Y}^{T} \boldsymbol{P} \boldsymbol{X}-\alpha \boldsymbol{V} & \boldsymbol{Y}^{T} \boldsymbol{P} \boldsymbol{Y}-\beta \boldsymbol{I} & \boldsymbol{Y}^{T} \boldsymbol{P} \boldsymbol{Z} \\
\boldsymbol{Z}^{T} \boldsymbol{P} \boldsymbol{X} & \boldsymbol{Z}^{T} \boldsymbol{P} \boldsymbol{Y} & \boldsymbol{Z}^{T} \boldsymbol{P} \boldsymbol{Z}-\gamma \boldsymbol{Q}_{\boldsymbol{v}}
\end{array}\right] \overline{\boldsymbol{v}}_{k}<\mathbf{0} .
$$

where $\boldsymbol{S}_{2}=\boldsymbol{X}^{T} \boldsymbol{P} \boldsymbol{X}-\boldsymbol{P}+\gamma \boldsymbol{P}+\alpha \boldsymbol{V}^{T}\left(\boldsymbol{M}+\boldsymbol{M}^{T}\right) \boldsymbol{V}+\beta \boldsymbol{V}^{T} \boldsymbol{M}^{T} \boldsymbol{M} \boldsymbol{V}$.

Applying Schur complements [60] to (31) yields

$$
\left[\begin{array}{cccc}
\boldsymbol{S}_{3} & -\alpha \boldsymbol{V}^{T} & \mathbf{0} & \boldsymbol{X}^{T} \boldsymbol{P} \\
-\alpha \boldsymbol{V} & -\beta \boldsymbol{I} & \mathbf{0} & \boldsymbol{Y}^{T} \boldsymbol{P} \\
\mathbf{0} & \mathbf{0} & -\gamma \boldsymbol{Q}_{\boldsymbol{v}} & \boldsymbol{Z}^{T} \boldsymbol{P} \\
\boldsymbol{P} \boldsymbol{X} & \boldsymbol{P} \boldsymbol{Y} & \boldsymbol{P} \boldsymbol{Z} & -\boldsymbol{P}
\end{array}\right] \prec \mathbf{0}
$$

where $\boldsymbol{S}_{3}=-\boldsymbol{P}+\gamma \boldsymbol{P}+\alpha \boldsymbol{V}^{T}\left(\boldsymbol{M}+\boldsymbol{M}^{T}\right) \boldsymbol{V}+\beta \boldsymbol{V}^{T} \boldsymbol{M}^{T} \boldsymbol{M} \boldsymbol{V}$. Applying Lemma 1 to 32 and then substituting

$$
\begin{aligned}
& P X=P \bar{A}-P \bar{K} \bar{C}=P \bar{A}-H \bar{C}, \\
& P Z=P \bar{W}-P \bar{K} \bar{V}=P \bar{W}-H \bar{V},
\end{aligned}
$$

where $\boldsymbol{H}=\boldsymbol{P} \overline{\boldsymbol{K}}$, lead to (22), which completes the proof.

\section{Bounded-error estimation}

This section provides a computational framework that can be used for obtaining a feasible state and fault set that is consistent with the current measurements. To solve the above problem, let us start with the following theorem: 
Theorem 2. If the system (16) is strictly quadratically bounded for all $\boldsymbol{v}_{k} \in \mathcal{E}_{\boldsymbol{v}}$, then the uncertainty intervals for the state and fault are given as follows:

$$
\begin{aligned}
& \hat{\boldsymbol{x}}_{i, k}-z_{i, k} \leq \boldsymbol{x}_{i, k} \leq \hat{\boldsymbol{x}}_{i, k}+z_{i, k}, \quad i=1, \ldots, n . \\
& \hat{\boldsymbol{f}}_{j, k}-z_{i, k} \leq f_{j, k} \leq \hat{\boldsymbol{f}}_{j, k}+z_{i, k}, \quad j=1, \ldots, r, \quad i=n+1, \ldots, n+r .
\end{aligned}
$$

with

$$
\begin{aligned}
z_{i, k} & =\left(\zeta_{k}(\gamma) \boldsymbol{c}_{i}^{T} \boldsymbol{P}^{-1} \boldsymbol{c}_{i}\right)^{\frac{1}{2}} \\
\zeta_{k}(\gamma) & =(1-\gamma)^{k} V_{0}+1-(1-\gamma)^{k}, \quad k=0,1, \ldots
\end{aligned}
$$

where $V_{k}=\overline{\boldsymbol{e}}_{k}^{T} \boldsymbol{P} \overline{\boldsymbol{e}}_{k}$ and $\boldsymbol{c}_{i}$ is the ith column of an $n+r$ order identity matrix.

Proof. Theorem 1 guarantees that there exist $\gamma \in(0,1)$ and $\boldsymbol{P} \succ \mathbf{0}$ such that (34) holds. Moreover, from (24) and (31) it can be shown that for all $M \in \mathbb{M}$

$$
\begin{aligned}
V_{k+1} & <(1-\gamma) V_{k}-\alpha\left(\overline{\boldsymbol{e}}_{k}^{T} \boldsymbol{V}^{T}\left(\boldsymbol{M}+\boldsymbol{M}^{T}\right) \boldsymbol{V} \overline{\boldsymbol{e}}_{k}-\boldsymbol{s}_{k}^{T} \boldsymbol{V} \overline{\boldsymbol{e}}_{k}-\overline{\boldsymbol{e}}_{k}^{T} \boldsymbol{V}^{T} \boldsymbol{s}_{k}\right) \\
& -\beta\left(\overline{\boldsymbol{e}}_{k}^{T} \boldsymbol{V}^{T} \boldsymbol{M}^{T} \boldsymbol{M} \boldsymbol{V} \overline{\boldsymbol{e}}_{k}-\boldsymbol{s}_{k}^{T} \boldsymbol{s}_{k}\right)+\gamma \boldsymbol{v}_{k}^{T} \boldsymbol{Q}_{v} \boldsymbol{v}_{k},
\end{aligned}
$$

Subsequently, by 25] and (28) as well as by the fact that $\boldsymbol{v}_{k}^{T} \boldsymbol{Q}_{v} \boldsymbol{v}_{k} \leq 1$, the upper bound of $V_{k+1}$ defined by 37 can be overbounded with the non-strict inequality of the form

$$
V_{k+1} \leq \gamma+(1-\gamma) V_{k}
$$

Following [56], by induction, inequality (38) yields

$$
V_{k} \leq \zeta_{k}(\gamma), \quad k=0,1, \ldots,
$$

where the sequence $\zeta_{k}(\gamma)$ is defined by (36). Thus, from $(39)$ it is evident that for any $\boldsymbol{v}_{k} \in \mathcal{E}_{\boldsymbol{v}}, \overline{\boldsymbol{e}}_{k}$ is contained inside the ellipsoid

$$
\overline{\boldsymbol{e}}_{k}^{T} \boldsymbol{P} \overline{\boldsymbol{e}}_{k} \leq \zeta_{k}(\gamma)
$$

The maximum and minimum values of $\overline{\boldsymbol{e}}_{i, k}$ can be computed by maximizing/minimizing $\boldsymbol{c}_{i}^{T} \overline{\boldsymbol{e}}_{k}$ under 40 . Using the Lagrange approach, the following Lagrange function can be formulated

$$
\boldsymbol{h}\left(\overline{\boldsymbol{e}}_{k}, \lambda\right)=\boldsymbol{c}_{i}^{T} \overline{\boldsymbol{e}}_{k}+\lambda\left(\overline{\boldsymbol{e}}_{k}^{T} \boldsymbol{P} \overline{\boldsymbol{e}}_{k}-\zeta_{k}(\gamma)\right),
$$


where $\lambda$ stands for the Lagrange multiplier. Differentiating (41) with respect to $\bar{e}_{k}$ and $\lambda$ yields

$$
\begin{aligned}
& \frac{\partial \boldsymbol{h}\left(\overline{\boldsymbol{e}}_{k}, \lambda\right)}{\partial \overline{\boldsymbol{e}}_{k}}=\boldsymbol{c}_{i}^{T}+2 \lambda \overline{\boldsymbol{e}}_{k}^{T} \boldsymbol{P}=\mathbf{0}, \\
& \frac{\partial \boldsymbol{h}\left(\overline{\boldsymbol{e}}_{k}, \lambda\right)}{\partial \lambda}=\overline{\boldsymbol{e}}_{k}^{T} \boldsymbol{P} \overline{\boldsymbol{e}}_{k}-\zeta_{k}(\gamma)=0 .
\end{aligned}
$$

Thus, from 42 , it can be shown that

$$
\overline{\boldsymbol{e}}_{k}^{T}=-\frac{1}{2 \lambda} \boldsymbol{c}_{i}^{T} \boldsymbol{P}^{-1}
$$

Substituting (44) into (43) leads to

$$
\lambda= \pm \frac{1}{2}\left(\zeta_{k}(\gamma)^{-1} \boldsymbol{c}_{i}^{T} \boldsymbol{P}^{-1} \boldsymbol{c}_{i}\right)^{\frac{1}{2}} .
$$

Finally, introducing (45) into (44) yields

$$
-z_{i, k} \leq \overline{\boldsymbol{e}}_{i, k} \leq z_{i, k}, \quad i=1, \ldots, n+r .
$$

where $z_{i, k}$ is given by (35), which completes the proof.

Remark 1. It can be easily shown that (36) can be written as

$$
\zeta_{k}(\gamma)=(1-\gamma)^{k}\left(V_{0}-1\right)+1, \quad k=0,1, \ldots,
$$

which for $\gamma \in(0,1)$ converges exponentially to one. Moreover, the convergence ratio depends on $\gamma$, i.e., a larger $\gamma$ yields a faster convergence. It should also be noted that the computation of $\zeta_{k}(\gamma)$ requires the knowledge of initial estimation error $\overline{\boldsymbol{e}}_{0}$, i.e., $V_{0}=\overline{\boldsymbol{e}}_{0}^{T} \boldsymbol{P} \overline{\boldsymbol{e}}_{0}$. However this can be relaxed by assuming that $V_{0} \leq \delta, \delta>0$, which boils down to

$$
\zeta_{k}(\gamma)=(1-\gamma)^{k}(\delta-1)+1, \quad k=0,1, \ldots,
$$

\section{Final design procedure}

This section presents the final design procedure for the proposed boundederror estimator. As it was already mentioned in the previous section, $\zeta_{k}$ converges exponentially to one, with a speed that depends on $\gamma$. Thus, the steady 
state length of (33) and (49) depends solely on the eigenvalues of $\boldsymbol{P}$, which describe the size of the ellipsoid. This means that, under constraints 22, the following functions can be employed:

1. D - optimality criterion $\phi(\boldsymbol{P})=\operatorname{det}(\boldsymbol{P})$

2. E - optimality criterion $\phi(\boldsymbol{P})=\lambda_{\max }(\boldsymbol{P})$

3. A - optimality criterion $\phi(\boldsymbol{P})=\operatorname{trace}(\boldsymbol{P})$

The determinant of $\boldsymbol{P}$ corresponds to the volume of the ellipsoid, while $\lambda_{\max }(\boldsymbol{P})$ stands for its largest axis. Finally, the trace of $\boldsymbol{P}$ corresponds to the sum of the axes of the ellipsoid. In the context of the approach developed in this paper, the A - optimality criterion is selected, such that the following optimization problem is obtained

$$
\max \operatorname{trace}(\boldsymbol{P}) \text {. }
$$

Having the cost function, it is desirable to describe $(22)$ in the linear matrix inequality (LMI) form.

Remark 2. The set $\mathbb{M}$, defined by (6), can be equivalently described by

$$
\mathbb{M}=\left\{\boldsymbol{M}(\alpha): \boldsymbol{M}(\alpha)=\sum_{i=1}^{N} \alpha_{i} \boldsymbol{M}_{i}, \sum_{i=1}^{N} \alpha_{i}=1, \alpha_{i} \geq 0\right\}
$$

where $N=2^{n^{2}}$. Note that this is a general description, which does not take into account that some elements of $\boldsymbol{M}$ may be constant. In such cases, $N$ is given by $N=2^{(n-c)^{2}}$, where c stands for the number of constant elements of $\boldsymbol{M}$. Thus, solving 49) under 22 with respect to $\boldsymbol{H}, \boldsymbol{U}, \boldsymbol{P}, \gamma, \alpha, \beta$ is equivalent to $\max \operatorname{trace}(\boldsymbol{P})$ subject to $($ for $i=1, \ldots, N)$

$\left[\begin{array}{ccccc}\boldsymbol{S}_{1} & -\alpha \boldsymbol{V}^{T} & \mathbf{0} & \overline{\boldsymbol{A}}^{T} \boldsymbol{P}-\overline{\boldsymbol{C}}^{T} \boldsymbol{H}^{T} & \boldsymbol{V}^{T} \boldsymbol{M}_{i}^{T} \boldsymbol{U}^{T} \\ -\alpha \boldsymbol{V} & -\beta \boldsymbol{I} & \mathbf{0} & \boldsymbol{Y}^{T} \boldsymbol{P} & \mathbf{0} \\ \mathbf{0} & \mathbf{0} & -\gamma \boldsymbol{Q}_{\boldsymbol{v}} & \overline{\boldsymbol{W}}^{T} \boldsymbol{P}-\overline{\boldsymbol{V}}^{T} \boldsymbol{H}^{T} & \mathbf{0} \\ \boldsymbol{P} \overline{\boldsymbol{A}}-\boldsymbol{H} \overline{\boldsymbol{C}} & \boldsymbol{P} \boldsymbol{Y} & \boldsymbol{P} \overline{\boldsymbol{W}}-\boldsymbol{H} \overline{\boldsymbol{V}} & -\boldsymbol{P} & \mathbf{0} \\ \boldsymbol{U} \boldsymbol{M}_{i} \boldsymbol{V} & \mathbf{0} & \mathbf{0} & \mathbf{0} & \beta \boldsymbol{I}-\boldsymbol{U}-\boldsymbol{U}^{T}\end{array}\right] \prec \mathbf{0}$,

and then determining $\overline{\boldsymbol{K}}=\boldsymbol{P}^{-1} \boldsymbol{H}$. 
Remark 3. The problem of solving (51) boils down to bilinear matrix inequalities, due to the product of $\gamma$ and $\boldsymbol{P}$ in $\boldsymbol{S}_{1}$. However, by fixing a value for $\gamma$, (51) becomes a set of LMIs. Since $\gamma$ is constrained to belong to the interval $(0,1)$, an iterative algorithm can be easily implemented.

Finally, it should be underlined that when (51) is feasible, then all its diagonal elements should be negative definite. In particular,

$$
-\boldsymbol{P}+\gamma \boldsymbol{P}+\alpha \boldsymbol{V}^{T}\left(\boldsymbol{M}_{i}+\boldsymbol{M}_{i}^{T}\right) \boldsymbol{V}^{T} \prec \mathbf{0} .
$$

Thus, when $\boldsymbol{V}^{T}\left(\boldsymbol{M}_{i}+\boldsymbol{M}_{i}^{T}\right) \boldsymbol{V}^{T} \prec \mathbf{0}$, then it is evident that the feasibility of (51) can be obtained more easily than in the opposite case. However, when $\boldsymbol{V}^{T}\left(\boldsymbol{M}_{i}+\boldsymbol{M}_{i}^{T}\right) \boldsymbol{V}^{T} \succ \mathbf{0}$, then the optimization procedure will select $\alpha$ sufficiently close to zero in order to tackle this unappealing effect. A similar property underlines the one-sided Lipschitz condition-based design procedure proposed in [50]. However, as it was already mentioned, the proposed approach is perceived as a generalisation of the former one.

To summarize, the following flowchart of the developed algorithm is proposed:

Determine $\boldsymbol{E}$ by using $(13)$ and then set $\boldsymbol{T}=\boldsymbol{I}+\boldsymbol{E} \boldsymbol{C}$ and $\boldsymbol{G}=\boldsymbol{T} \boldsymbol{B}$

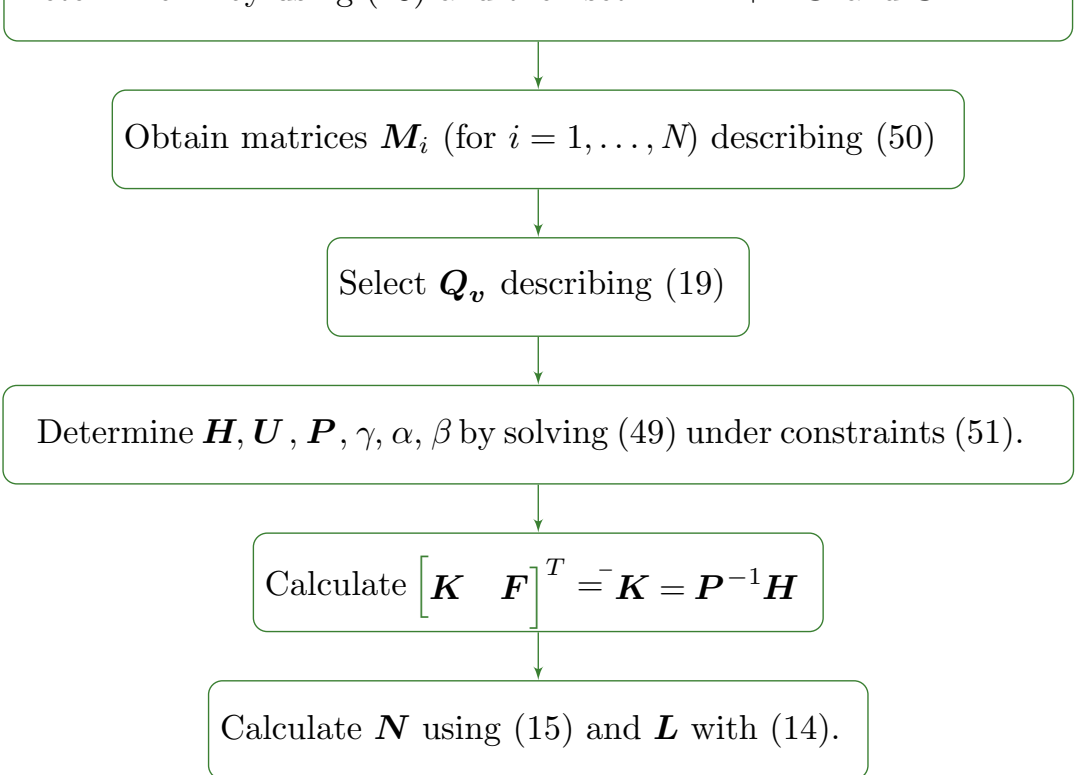




\section{Illustrative example}

Let us consider a quadruple-tank system [61, as depicted in Fig. 1. The dynamics of this system can be derived from mass balances and Bernoulli's law [62, yielding

$$
\begin{aligned}
& \frac{d h_{1}}{d t}=-\frac{a_{1}}{A_{1}} \sqrt{2 g h_{1}}+\frac{a_{3}}{A_{1}} \sqrt{2 g h_{3}}+\frac{\gamma_{1} k_{1}}{A_{1}} v_{1} \\
& \frac{d h_{2}}{d t}=-\frac{a_{2}}{A_{2}} \sqrt{2 g h_{2}}+\frac{a_{4}}{A_{2}} \sqrt{2 g h_{4}}+\frac{\gamma_{2} k_{2}}{A_{2}} v_{2} \\
& \frac{d h_{3}}{d t}=-\frac{a_{3}}{A_{3}} \sqrt{2 g h_{3}}+\frac{\left(1-\gamma_{2}\right) k_{2}}{A_{3}} v_{2} \\
& \frac{d h_{4}}{d t}=-\frac{a_{4}}{A_{4}} \sqrt{2 g h_{4}}+\frac{\left(1-\gamma_{1}\right) k_{1}}{A_{4}} v_{1}
\end{aligned}
$$

where $A_{i}, a_{i}$ and $h_{i}, i=1,2,3,4$, denote the cross-section of tanks, the crosssections of the outlet holes and the water levels, respectively. The voltage applied to pump $i, i=1,2$ is $v_{i}$, and the corresponding flow is $k_{i} v_{i}$. The acceleration of gravity is denoted by $g$. The parameter values are given by: $A_{1}=$ $A_{3}=28 \mathrm{~cm}^{2}, A_{2}=A_{4}=32 \mathrm{~cm}^{2}, a_{1}=a_{3}=0.071 \mathrm{~cm}^{2}, a_{2}=a_{4}=0.051 \mathrm{~cm}^{2}$, $k_{1}=3.235 \mathrm{~cm}^{3} / V s, k_{2}=3.320 \mathrm{~cm}^{3} / V s, \gamma_{1}=0.5650$ and $\gamma_{2}=0.47$.

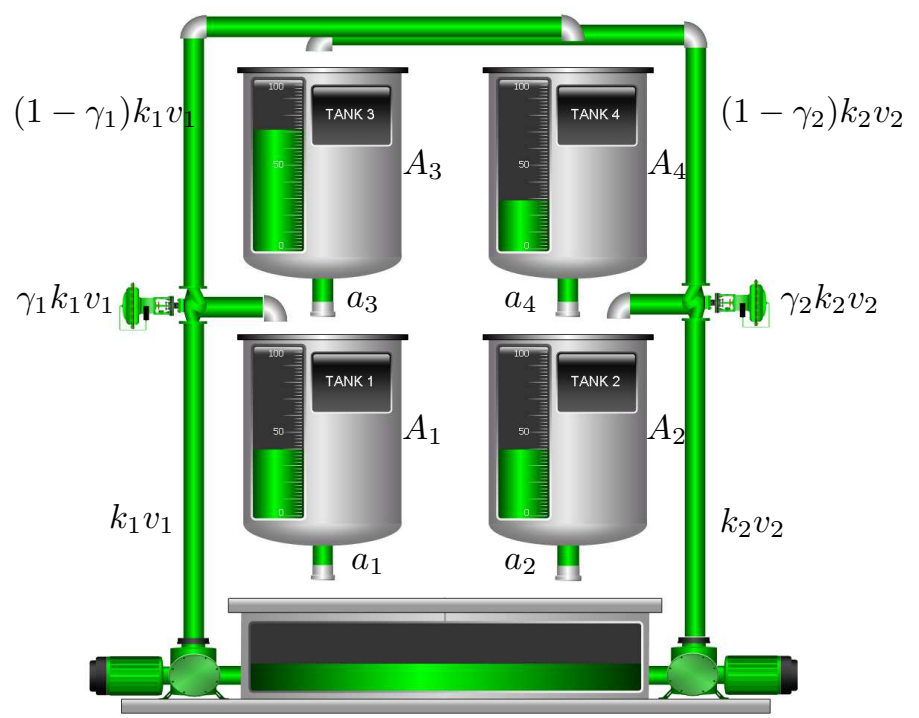

Figure 1: Quadruple-tank system 
By defining $\boldsymbol{x}=\left[h_{1}, h_{2}, h_{3}, h_{4}\right]^{T}$ and $\boldsymbol{u}=\left[v_{1}, v_{2}\right]^{T}$, the nonlinear model 53)-56 can be reshaped in the quasi-linear form

$$
\begin{gathered}
\dot{\boldsymbol{x}}=\boldsymbol{A}_{Q L}(\boldsymbol{x}) \boldsymbol{x}+\boldsymbol{B}_{Q L} \boldsymbol{u} \\
\boldsymbol{A}_{Q L}(\boldsymbol{x})=\left[\begin{array}{cccc}
-\frac{a_{1} \sqrt{2 g h_{1}}}{A_{1} h_{1}} & 0 & \frac{a_{3} \sqrt{2 g h_{3}}}{A_{1} h_{3}} & 0 \\
0 & -\frac{a_{2} \sqrt{2 g h_{2}}}{A_{2} h_{2}} & 0 & \frac{a_{4} \sqrt{2 g h_{4}}}{A_{2} h_{4}} \\
0 & 0 & -\frac{a_{3} \sqrt{2 g h_{3}}}{A_{3} h_{3}} & 0 \\
0 & 0 & 0 & -\frac{a_{4} \sqrt{2 g h_{4}}}{A_{4} h_{4}}
\end{array}\right] \\
\boldsymbol{B}_{Q L}=\left[\begin{array}{cc}
\frac{\gamma_{1} k_{1}}{A_{1}} & 0 \\
0 & \frac{\gamma_{2} k_{2}}{A_{2}} \\
0 & \frac{\left(1-\gamma_{2}\right) k_{2}}{A_{3}} \\
\frac{\left(1-\gamma_{1}\right) k_{1}}{A_{4}} & 0
\end{array}\right]
\end{gathered}
$$

Eq. (57) is discretized using Euler method with sampling time $T_{s}=1 \mathrm{~s}$, such that it can be expressed as (1)-(2) around an equilibrium point $\boldsymbol{x}_{e q}$, as follows

$$
\begin{aligned}
& \boldsymbol{x}_{k+1}=\boldsymbol{A}\left(\boldsymbol{x}_{e q}\right) \boldsymbol{x}_{k}+\boldsymbol{B}\left(\boldsymbol{x}_{e q}\right) \boldsymbol{u}_{k}+\boldsymbol{D} \boldsymbol{d}_{k}+\boldsymbol{g}\left(\boldsymbol{x}_{e q}, \boldsymbol{x}_{k}, \boldsymbol{u}_{k}\right)+\boldsymbol{B}\left(\boldsymbol{x}_{e q}\right) \boldsymbol{f}_{k}+\boldsymbol{W}_{1} \boldsymbol{w}_{k}, \\
& \boldsymbol{y}_{k}=\boldsymbol{C} \boldsymbol{x}_{k}+\boldsymbol{W}_{2} \boldsymbol{w}_{k}
\end{aligned}
$$

where $\boldsymbol{A}\left(\boldsymbol{x}_{e q}\right)$ and $\boldsymbol{B}\left(\boldsymbol{x}_{e q}\right)$ are the frozen system matrices at the equilibrium point, while the non-linear function $\boldsymbol{g}\left(\boldsymbol{x}_{e q}, \boldsymbol{x}_{k}, \boldsymbol{u}_{k}\right)$ is defined as

$$
\boldsymbol{g}\left(\boldsymbol{x}_{e q}, \boldsymbol{x}_{k}, \boldsymbol{u}_{k}\right)=\left(\boldsymbol{A}\left(\boldsymbol{x}_{k}\right)-\boldsymbol{A}\left(\boldsymbol{x}_{e q}\right)\right) \boldsymbol{x}_{k}
$$

In particular, for input signals $\boldsymbol{u}_{k}=[10,10]^{T}$, the equilibrium point $\boldsymbol{x}_{e q}=$ $[130.1,172.6,31.3,38.8]^{T}$ can be calculated, which corresponds to matrices $\boldsymbol{A}\left(\boldsymbol{x}_{e q}\right)$ and $\boldsymbol{B}$ as follows

$$
\boldsymbol{A}\left(\boldsymbol{x}_{e q}\right)=\left[\begin{array}{cccc}
0.9902 & 0 & 0.0201 & 0 \\
0 & 0.9946 & 0 & 0.0113 \\
0 & 0 & 0.9799 & 0 \\
0 & 0 & 0 & 0.9887
\end{array}\right], \boldsymbol{B}=\left[\begin{array}{cc}
0.0653 & 0 \\
0 & 0.0488 \\
0 & 0.0628 \\
0.0440 & 0
\end{array}\right] \text {. }
$$


It will be assumed that $h_{1}, h_{2}$ and $h_{3}$ are directly measured, such that

$$
\boldsymbol{C}=\left[\begin{array}{llll}
1 & 0 & 0 & 0 \\
0 & 1 & 0 & 0 \\
0 & 0 & 1 & 0
\end{array}\right],
$$

and that the exogenous disturbance $\boldsymbol{w}_{k}$ affects the output equation only, such that $\boldsymbol{W}_{1}=\mathbf{0}$ and $\boldsymbol{W}_{2}=\boldsymbol{I}_{3}$. On the other hand, in order to show the decoupling property of the proposed strategy, an unknown input acting on the third tank is considered, i.e. $\boldsymbol{D}=[0,0,1,0]^{T}$.

By varying each component of $\boldsymbol{x}_{k}$ within its possible domain, which is assumed to be $h_{1}, h_{2} \in[60,280]$ and $h_{3}, h_{4} \in[5,100]$, it is possible to obtain the matrices

$$
\begin{gathered}
\boldsymbol{M}_{\max }=\left[\begin{array}{cccc}
0.0065 & 0 & 0.0039 & 0 \\
0 & 0.0033 & 0 & 0.0037 \\
0 & 0 & 0.0144 & 0 \\
0 & 0 & 0 & 0.0078
\end{array}\right], \\
\boldsymbol{M}_{\min }=\left[\begin{array}{cccc}
0.0026 & 0 & -0.0144 & 0 \\
0 & 0.0008 & 0 & -0.0078 \\
0 & 0 & -0.0039 & 0 \\
0 & 0 & 0 & -0.0037
\end{array}\right] .
\end{gathered}
$$

which contain all elements $\bar{a}_{i, j}$ and $\underline{a}_{i, j}, i, j=1, \ldots, n$ defining (6), respectively. Afterwards, taking into account all the possible combinations of elements in $\boldsymbol{M}_{\max }$ and $\boldsymbol{M}_{\min }, 64$ matrices $\boldsymbol{M}_{i}$ are obtained (see [51)). Let the initial condition for the system and the observer be

$$
\boldsymbol{x}_{0}=[130,170,30,40]^{T}, \boldsymbol{z}_{0}=[125,165,25,35]^{T}, \hat{\boldsymbol{f}_{0}}=\mathbf{0}
$$

while the input

$$
v_{1}(k)=10+\sin \left(\frac{k}{50}\right), \quad v_{2}(k)=10+\cos \left(\frac{k}{80}\right)
$$

and constant input disturbance $d_{k}=0.1$ are considered, and $\boldsymbol{w}_{k}$ is chosen as 225 a uniformly distributed random vector, where each element takes values in the 
interval $[-0.1,0.1]$, which corresponds to $\boldsymbol{Q}_{w}=100 \boldsymbol{I}_{3}$. Let us consider the following fault scenarios, containing additive faults in both pump 1 and pump 2 , characterized as follows

\section{Fault scenario FS1:}

$$
\begin{aligned}
& f_{1}(k)=\left\{\begin{array}{cc}
0 & 0 \leq k \leq 1000 \\
-0.05 k+50 & 1000<k \leq 1100 \\
-5 & 1100<k \leq 3000 \\
0.05 k-155 & 3000<k \leq 3200 \\
5 & \text { otherwise }
\end{array}\right. \\
& f_{2}(k)=0
\end{aligned}
$$

\section{Fault scenario FS2:}

$$
\begin{gathered}
f_{1}(k)=\left\{\begin{array}{cc}
0 & 0 \leq k \leq 1000, \\
-0.05 k+50 & 1000<k \leq 1100, \\
-5 & 1100<k \leq 3000, \\
0.05 k-155 & 3000<k \leq 3200, \\
5 & \text { otherwise }
\end{array}\right. \\
f_{2}(k)=\left\{\begin{array}{cc}
0 \leq k \leq 1000, \\
0 & 1000<k \leq 1100, \\
0.05 k-50 & 1100<k \leq 3000, \\
5 & 3000<k \leq 3200, \\
-0.05 k+155 & \text { otherwise. }
\end{array}\right.
\end{gathered}
$$

Once $\gamma=0.01$ is chosen, LMIs (51) can be solved maximizing trace $(\boldsymbol{P})$ and obtaining the following matrices

$$
\boldsymbol{N}=\left[\begin{array}{cccc}
0.6315 & 0.0275 & 0.0793 & 0 \\
0.0262 & 0.7383 & -0.0053 & 0.0113 \\
-8.6 \cdot 10^{-6} & -2.1 \cdot 10^{-6} & 4.8 \cdot 10^{-5} & 0 \\
-0.2542 & 0.0278 & 0.0511 & 0.9887
\end{array}\right], \quad \boldsymbol{F}^{T}=\left[\begin{array}{cc}
0.6107 & -0.0632 \\
-0.0306 & 0.5335 \\
-0.1255 & 0.0129
\end{array}\right]
$$




$$
\begin{gathered}
\boldsymbol{G}=\left[\begin{array}{cc}
0.0653 & 0 \\
0 & 0.0488 \\
0 & 0 \\
0.0440 & 0
\end{array}\right], \quad \boldsymbol{L}=\left[\begin{array}{ccc}
0.3587 & -0.0275 & 0.0201 \\
-0.0262 & 0.2563 & 0 \\
8.6 \cdot 10^{-6} & 2.1 \cdot 10^{-6} & 0 \\
0.2542 & -0.0278 & 0
\end{array}\right], \\
\boldsymbol{T}=\left[\begin{array}{cccc}
1 & 0 & 0 & 0 \\
0 & 1 & 0 & 0 \\
0 & 0 & 0 & 0 \\
0 & 0 & 0 & 1
\end{array}\right], \quad \boldsymbol{E}=\left[\begin{array}{ccc}
0 & 0 & 0 \\
0 & 0 & 0 \\
0 & 0 & -1 \\
0 & 0 & 0
\end{array}\right]
\end{gathered}
$$

Figs. 247 present the real values of each state/fault (black lines), their estimation (red lines) and the uncertainty intervals (green lines) obtained for the fault scenario FS1. In particular, in Figs. 25, the responses corresponding to a simulation which lasts $5000 \mathrm{~s}$ are plotted alongside with two zooms, that show the convergence of the estimation to the real value and the inclusion of the state within the uncertainty intervals. From these results, it is evident that the estimation is performed with a good quality. Similar results have been obtained in FS2, as shown in Figs. 813 , which proves the effectiveness of the proposed approach in dealing with multiple faults.

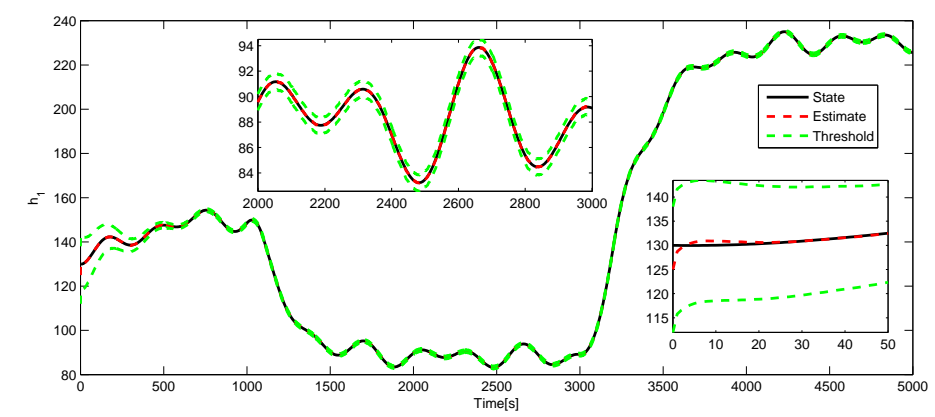

Figure 2: Tank level $h_{1}$ (black line), its estimate (red line) and its uncertainty intervals (green lines) for the fault scenario FS1 


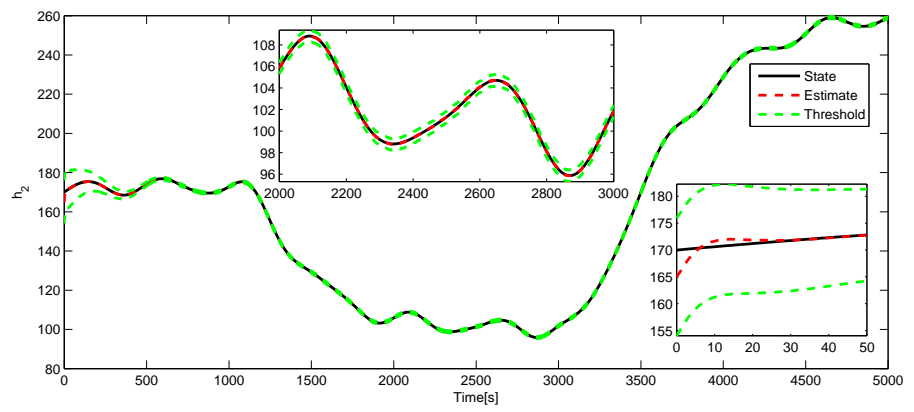

Figure 3: Tank level $h_{2}$ (black line), its estimate (red line) and its uncertainty intervals (green lines) for the fault scenario FS1

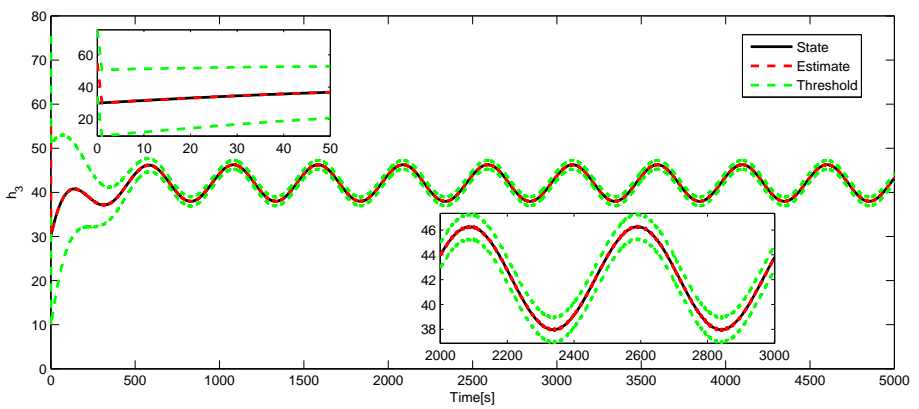

Figure 4: Tank level $h_{3}$ (black line), its estimate (red line) and its uncertainty intervals (green lines) for the fault scenario FS1

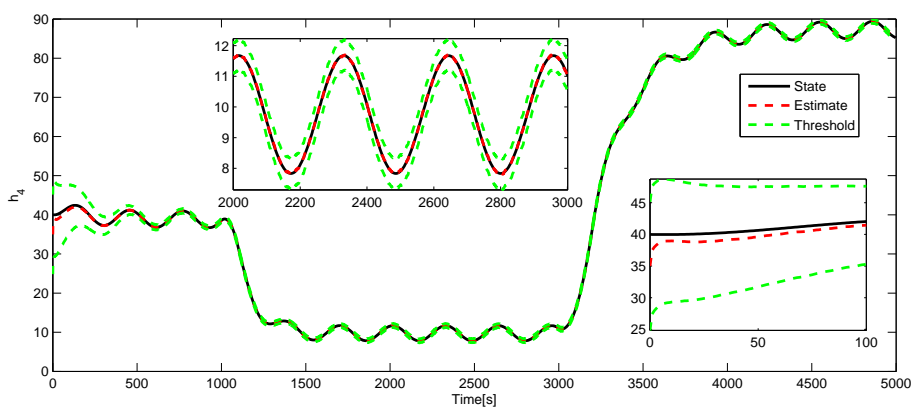

Figure 5: Tank level $h_{4}$ (black line), its estimate (red line) and its uncertainty intervals (green lines) for the fault scenario FS1 


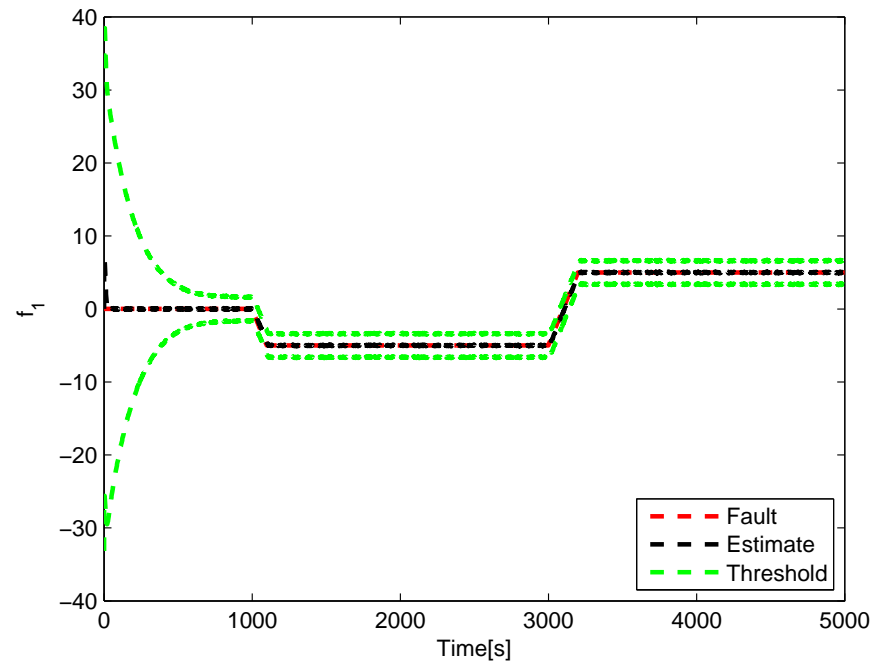

Figure 6: Actuator fault $f_{1}$ (black line), its estimate (red line) and its uncertainty intervals (green lines) for the fault scenario FS1

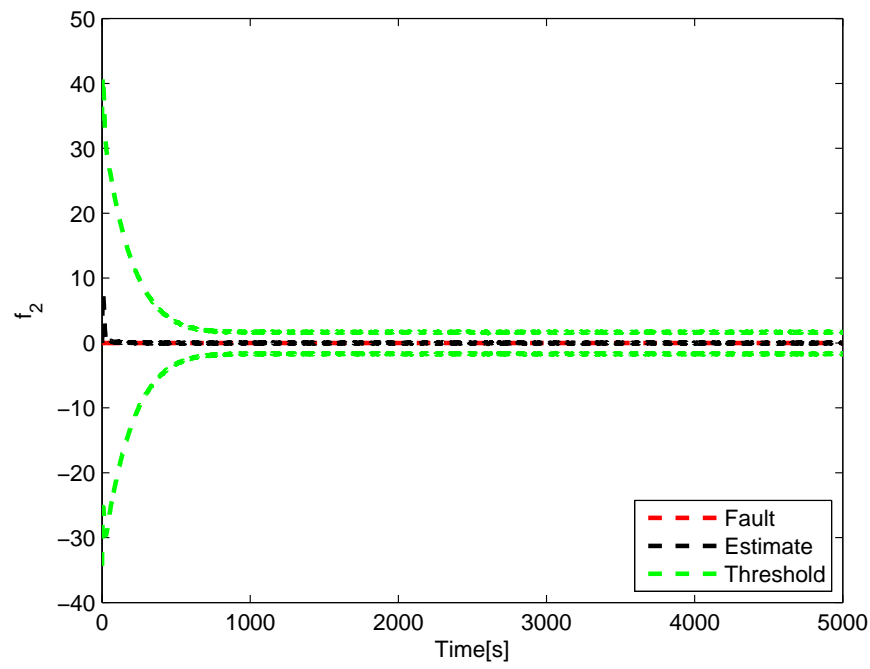

Figure 7: Actuator fault $f_{2}$ (black line), its estimate (red line) and its uncertainty intervals (green lines) for the fault scenario FS1 


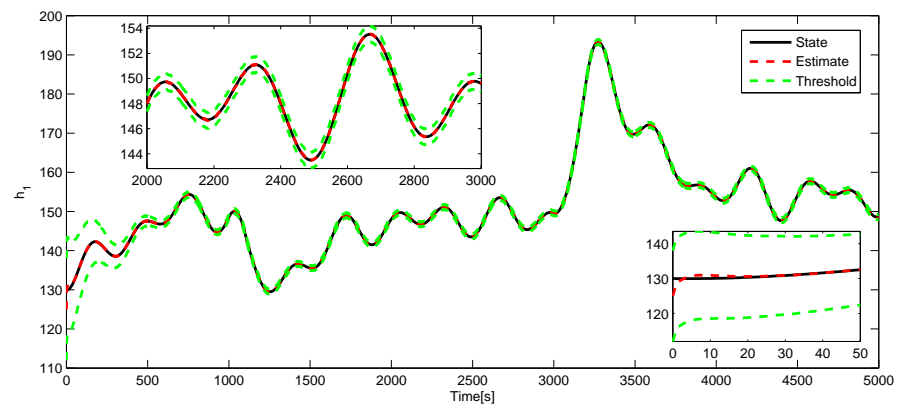

Figure 8: Tank level $h_{1}$ (black line), its estimate (red line) and its uncertainty intervals (green lines) for the fault scenario FS2

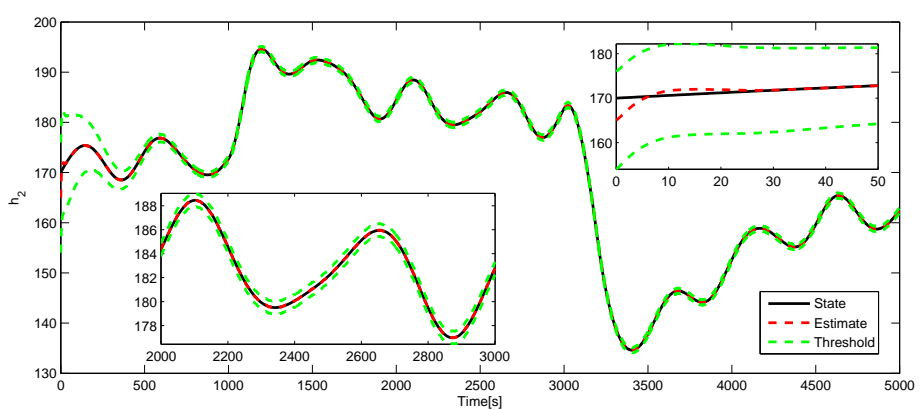

Figure 9: Tank level $h_{2}$ (black line), its estimate (red line) and its uncertainty intervals (green lines) for the fault scenario FS2

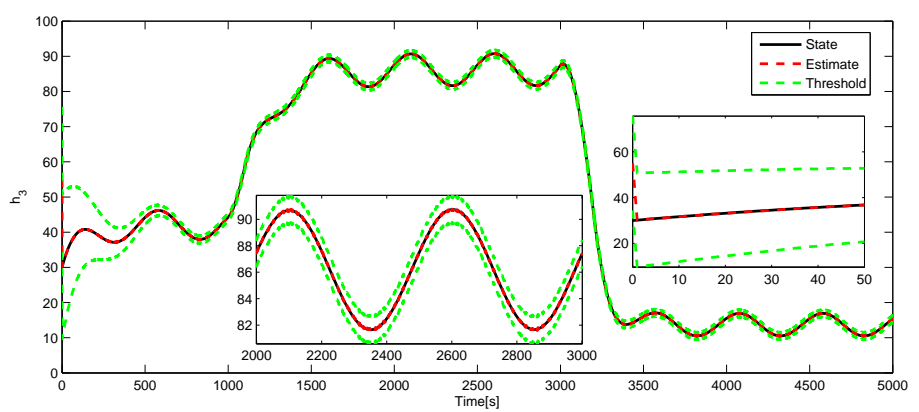

Figure 10: Tank level $h_{3}$ (black line), its estimate (red line) and its uncertainty intervals (green lines) for the fault scenario FS2 


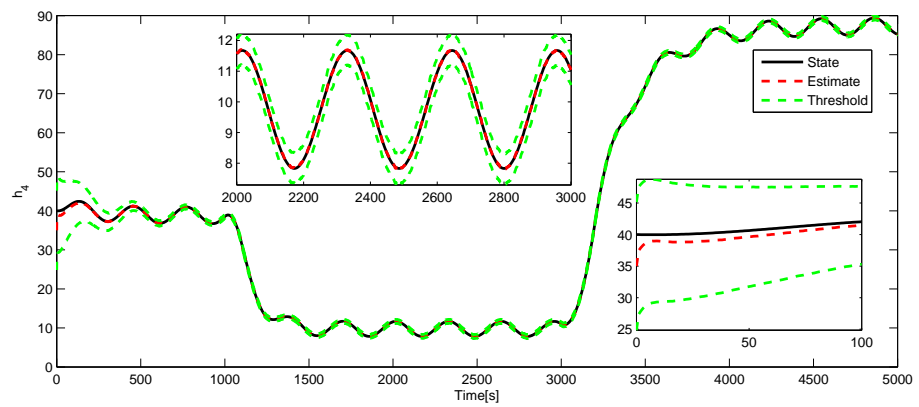

Figure 11: Tank level $h_{4}$ (black line), its estimate (red line) and its uncertainty intervals (green lines) for the fault scenario FS2

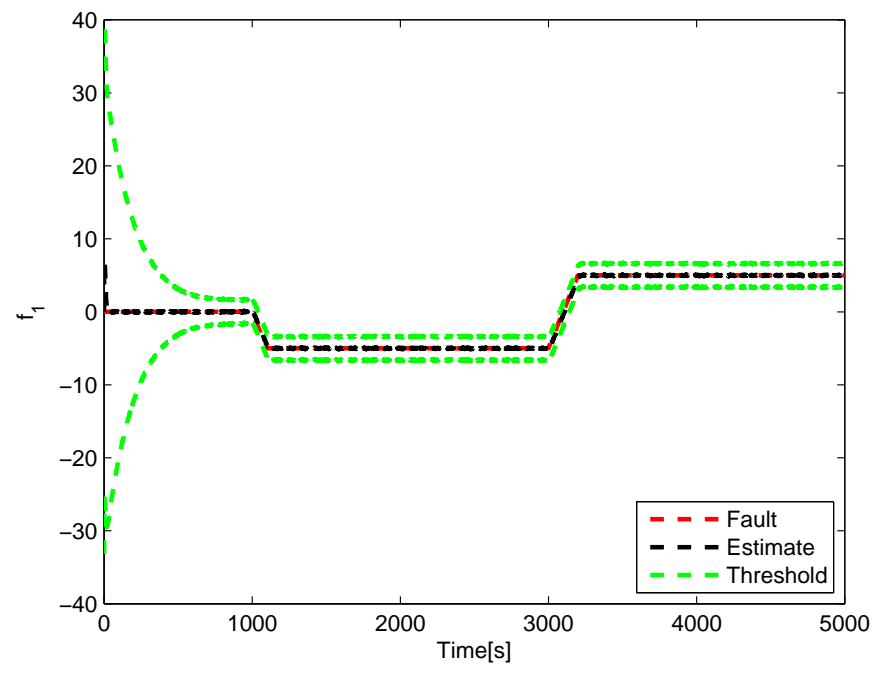

Figure 12: Actuator fault $f_{1}$ (black line), its estimate (red line) and its uncertainty intervals (green lines) for the fault scenario FS2

\section{Conclusions}

This paper has proposed an estimator for the simultaneous estimation of states and actuator faults in a class of nonlinear systems that satisfy a one-sided Lipschitz condition. The design approach for the estimator can be perceived as a set-membership one, since the theory of quadratic boundedness is used to 


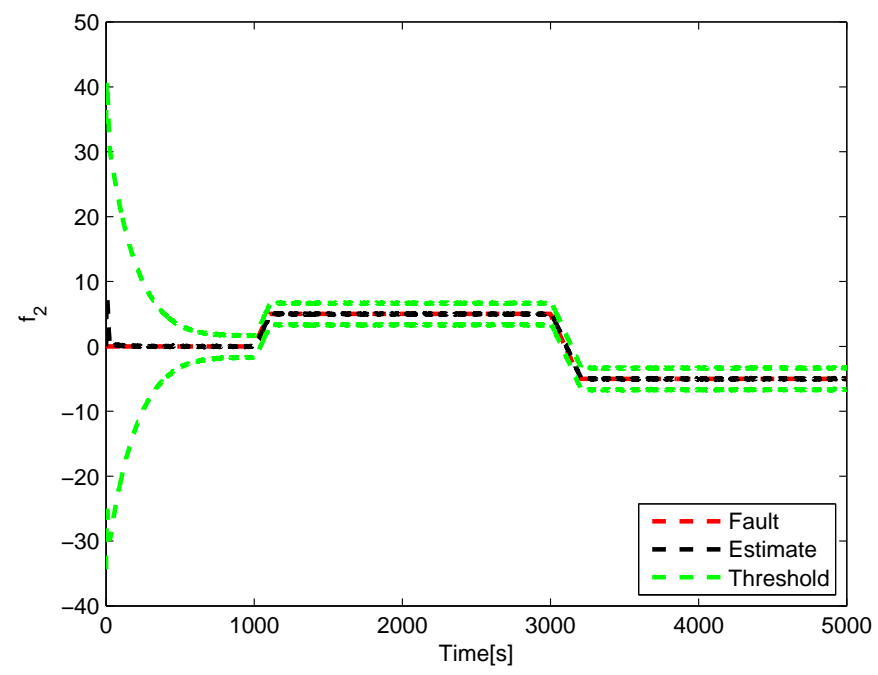

Figure 13: Actuator fault $f_{2}$ (black line), its estimate (red line) and its uncertainty intervals (green lines) for the fault scenario FS2

provide the set of states and faults that are consistent with the measurements, assuming that the external sources of uncertainty (disturbances and measurement noise) are unknown but bounded. Sufficient conditions for the existence and stability of the proposed state and actuator fault estimator have been obtained and expressed in the form of linear matrix inequalities (LMIs), which can be solved efficiently using available solvers. The application of the proposed approach to a quadruple-tank system has allowed assessing its effectiveness.

\section{Acknowledgments}

The authors would like to thank the reviewers for their valuable comments and suggestions. The work was supported by the National Science Centre of Poland under grant: 2013/11/B/ST7/01110 and the Research Council of Norway through the Centers of Excellence funding scheme, Project number 223254 - Centre for Autonomous Marine Operations and Systems (NTNUAMOS). D. Rotondo is also supported by the ERCIM Alain Bensoussan Fellow- 
ship programme. The work has also been funded by the Spanish Government (MINECO) through the project CICYT ECOCIS (ref. DPI2013-48243-C2-1-R) and by MINECO and FEDER through the project CICYT HARCRICS (ref. DPI2014-58104-R).

\section{References}

[1] C. D. Persis, R. D. Santis, A. Isidori, Nonlinear actuator fault detection and isolation for a VTOL aircraft, in: Proceedings of the 2001 American Control Conference, June 25-27, 2001, pp. 4449-4454.

[2] F. Amato, C. Cosentino, M. Mattei, G. Paviglianiti, A mixed direct/functional redundancy scheme for the FDI on a small commercial aircraft, in: Proceedings of the SAFEPROCESS03, IFAC symposium, 2003, pp. 331-339.

[3] B. Jiang, M. Staroswiecki, V. Cocquempot, Fault accommodation for nonlinear dynamic systems, IEEE Transactions on Automatic Control 51 (9) (2006) 1578-1583.

[4] M. Staroswiecki, G. Comtet-Varga, Analytical redundancy relations for fault detection and isolation in algebraic dynamic systems, Automatica 37 (5) (2001) 687-699.

[5] H. Hammouri, P. Kabore, S. Othman, J. Biston, Observer-based approach to fault detection and isolation for nonlinear systems, IEEE Transactions on Automatic Control 44 (10) (1999) 1879-1884.

[6] C. D. Persis, R. D. Santis, A. Isidori, An $\mathcal{H}_{\infty}$-suboptimal fault detection filter for bilinear systems, in: Proceedings of the Nonlinear control in the Year 2000, 2001, pp. 331-339.

[7] B.Jiang, J.L.Wang, Actuator fault diagnosis for a class of bilinear systems with uncertainty, Journal of the Franklin Institute 339 (2) (2002) 361-374. 
[8] R. Isermann, Fault Diagnosis Applications: Model Based Condition Monitoring, Actuators, Drives, Machinery, Plants, Sensors, and Fault-tolerant Systems, Springer-Verlag, Berlin, 2011.

[9] M. Mrugalski, An unscented Kalman filter in designing dynamic GMDH neural networks for robust fault detection, International Journal of Applied Mathematics and Computer Science 23 (1) (2013) 157-169.

[10] J. Korbicz, J. Kościelny, Z. Kowalczuk, W. Cholewa (Eds.), Fault Diagnosis. Models, Artificial Intelligence, Applications, Springer-Verlag, Berlin, 2012.

[11] Y. Liu, H. Wang, J. Yu, P. Li, Selective recursive kernel learning for online identification of nonlinear systems with NARX form, Journal of Process Control 20 (2) (2010) 181-194.

[12] W. Chen, A. Khan, M. Abid, S. Ding, Integrated design of observer-based fault detection for a class of uncertain non-linear systems., International Journal of Applied Mathematics and Computer Science 21 (4) (2011) 619636.

[13] T. Floquet, C. Edwards, S. K. Spurgeon, On sliding mode observer for systems with unknown inputs, International Journal of Adaptive Control and Signal Processing 21 (8) (2007) 638-656.

[14] V. Puig, Fault diagnosis and fault tolerant control using set-membership approaches: Application to real case studies, International Journal of Applied Mathematics and Computer Science 20 (4) (2010) 619-635.

305

[15] G. Calafiore, L. El Ghaoui, Ellipsoidal bounds for uncertain linear equations and dynamical systems, Automatica 40 (2004) 773-787.

[16] A. Hero, Y. Zhang, W. Rogers, Tomographic feature detection and classification using paralletolpe bounded error estimation, IEEE International Conference on Acoustics, Speech and Signal Processing 4 (1997) 2849-2852. 
[24] C. Edwards, S. K. Spurgeon, R. J. Patton, Sliding mode observers for fault detection and isolation, Automatica 36 (2000) 541-553.

[25] X. Zhang, M. Polycarpou, T. Prisini, Fault diagnosis of a class of nonlinear uncertain systems with Lipschitz nonlinearities using adaptive estimation,

[17] G. M. Ziegler, Lectures on polytopes, Graduate texts in mathematics, Springer, New York, 1995.

[18] M. Milanese, G. Belforte, Estimation theory and uncertainty intervals evaluation in presence of unknown but bounded errors: Linear families of models and estimators, IEEE Transaction on Automatic Control 27 (1992) 408414.

[19] J. Bravo, T. Alamo, M. Redondo, E. Camacho, An algorithm for boundederror identification of nonlinear systems based on dc functions., Automatica 44 (2008) 437-444.

[20] S. M. Tabatabaeipour, T. Bak, Robust observer-based fault estimation and accommodation of discrete-time piecewise linear systems, Journal of the Franklin Institute 351 (1) (2014) 277 - 295.

[21] S.-A. Raka, C. Combastel, Fault detection based on robust adaptive thresholds: A dynamic interval approach, Annual Reviews in Control 37 (1) (2013) 119-128.

[22] I. Fagarasana, S. Ploix, S. Gentil, Fault diagnosis and fault tolerant control using set-membership approaches: Application to real case studies, Automatica 40 (12) (2004) 2099-2110.

[23] J. Blesa, V. Puig, J. Saludes, Identification for passive robust fault detection using zonotope-based set-membership approaches, International Journal of Adaptive Control and Signal Processing 25 (2011) 788-812. Automatica 46 (2) (2010) 290-299. 
[26] F. López-Estrada, J. Ponsart, C. Astorga-Zaragoza, J. Camas-Anzueto, D. Theilliol, Robust sensor fault estimation for descriptor-lpv systems with unmeasurable gain scheduling functions: Application to an anaerobic bioreactor, International Journal of Applied Mathematics and Computer Science 25 (2) (2015) 233-244.

[27] J. Byrski, W. Byrski, A double window state observer for detection and isolation of abrupt changes in parameters, International Journal of Applied Mathematics and Computer Science 26 (3) (2016) 585-602.

[28] R. Patton, S. Klinkhieo, Actuator fault estimation and compensation based on an augmented state observer approach, in: Proceedings of the 48th IEEE Conference on Decision and Control, 2009 held jointly with the 2009 28th Chinese Control Conference. CDC/CCC 2009, Shanghai, China, IEEE, 2009, pp. 8482-8487.

[29] D. Rotondo, F. Nejjari, V. Puig, A virtual actuator and sensor approach for fault tolerant control of LPV systems, Journal of Process Control 24 (3) (2014) 203-222.

[30] M. M. Seron, J. A. De Doná, Robust fault estimation and compensation for LPV systems under actuator and sensor faults, Automatica 52 (2015) $294-301$.

[31] M. Witczak, M. Buciakowski, V. Puig, D. Rotondo, F. Nejjari, An LMI approach to robust fault estimation for a class of nonlinear systems, International Journal of Robust and Nonlinear Control 26 (7) (2015) 1530-1548.

[32] M. C. Nguyen, H. Trinh, Unknown input observer design for one-sided lipschitz discrete-time systems subject to time-delay, Applied Mathematics and Computation 286 (2016) 57-71.

[33] M. C. Nguyen, H. Trinh, Reduced-order observer design for one-sided lipschitz time-delay systems subject to unknown inputs, IET Control Theory \& Applications 10 (10) (2016) 1097-1105. 
[34] M. Aldeen, R. Sharma, Estimation of states, faults and unknown disturbances in non-linear systems, International Journal of Control 81 (8) (2008) 1195-1201.

[35] Z. Gao, S. X. Ding, Sensor fault reconstruction and sensor compensation for a class of nonlinear state-space systems via a descriptor system approach, IET Control Theory and Applications 1 (3) (2007) 578-585.

[36] G. Besancon, High-gain observation with disturbance attenuation and application to robust fault detection, Automatica 39 (6) (2003) 1095-1102.

[37] A. M. Pertew, H. J. Marquez, Q. Zhao, $\mathcal{H}_{\infty}$ synthesis of unknown input observers for non-linear Lipschitz systems, International Journal of Control 78 (15) (2005) 1155-1165.

[38] G. Hu, Observers for one-sided lipschitz non-linear systems, IMA Journal of Mathematical Control and Information 46 (2006) 395-401.

[39] A. Pertew, H. Marquez, Q. Zhao, LMI-based sensor fault diagnosis for nonlinear Lipschitz systems, Automatica 43 (8) (2007) 1464-1469.

[40] G. Hu, A note on observer for one-sided Lipschitz non-linear systems, IMA Journal of Mathematical Control and Information 25 (3) (2006) 297-303.

[41] Q. P. Ha, H. Trinh, State and input simultaneous estimation for a class of nonlinear systems, Automatica 40 (10) (2004) 1779-1785.

[42] H. Trinh, Q. P. Ha, State and input simultaneous estimation for a class of time-delay systems with uncertainties, IEEE Transactions on Circuits and Systems II: Express Briefs 54 (6) (2007) 527-531.

[43] J. Löfberg, Yalmip : a toolbox for modeling and optimization in matlab, in: Proceedings of the CACSD Conference, 2004, pp. 284-289.

[44] A. Zemouche, M. Boutayeb, Observer design for Lipschitz non-linear systems: the discrete time case, IEEE Transactions on Circuits and Systems - II:Express Briefs 53 (8) (2006) 777-781. 
[45] D. Stipanovic, D. Siljak, Robust stability and stabilization of discrete-time non-linear: the LMI approach, International Journal of Control 74 (5) (2001) 873-879.

[46] A. Zemouche, M. Boutayeb, G. Iulia Bara, Observer for a class of Lipschitz systems with extension to $\mathcal{H}_{\infty}$ performance analysis, Systems and Control Letters 57 (1) (2008) 18-27.

[47] M. Abbaszadeh, H. Marquez, LMI optimization approach to robust $\mathcal{H}_{\infty}$ observer design and static output feedback stabilization for non-linear uncertain systems, International Journal of Robust and Nonlinear Control 19 (3) (2008) 313-340.

[48] H. Marquez, Nonlinear control systems. Analysis and Design., Wiley, New Jersey, 2003.

[49] R. Rajamani, Observers for Lipschitz non-linear systems., IEEE Transactions on Automatic Control 43 (3) (1998) 397-401.

[50] Y. Zhao, J. Tao, N. Shi, A note on observer design for one-sided Lipschitz nonlinear systems, Systems and Control Letters 59 (1) (2010) 66-71.

[51] S. Gillijns, B. De Moor, Unbiased minimum-variance input and state estimation for linear discrete-time systems, Automatica 43 (2007) 111-116.

[52] M. Chadli, H. Karimi, Robust observer design for unknown inputs TakagiSugeno models, IEEE Transactions on Fuzzy Systems 21 (1) (2013) 158164.

[53] F. Schweppe, Uncertain dynamic systems, Prentice Hall, 1973.

[54] M. Blanke, M. Kinnaert, J. Lunze, M. Staroswiecki, Diagnosis and FaultTolerant Control, Second Edition. Springer-Verlag Berlin Heidelberg, 2006.

415 [55] M. Witczak, Fault Diagnosis and Fault-Tolerant Control Strategies for NonLinear Systems: Analytical and Soft Computing approaches, Springer International Publishing, Heidelberg, Germany, 2014. 
[56] A. Alessandri, M. Baglietto, G. Battistelli, Design of state estimators for uncertain linear systems using quadratic boundedness, Automatica 42 (3) (2006) 497-502.

[57] B. Ding, Constrained robust model predictive control via parameterdependent dynamic output feedback, Automatica 46 (9) (2010) 1517-1523.

[58] B. Ding, Dynamic output feedback predictive control for nonlinear systems represented by a Takagi-Sugeno model, Fuzzy Systems, IEEE Transactions on 19 (5) (2011) 831-843.

[59] M. de Oliveira, J. Bernussou, J. Geromel, A new discrete-time robust stability condition, Systems and Control Letters 37 (4) (1999) 261-265.

[60] S. Boyd, L. Ghaoui, E. Feron, V. Balakrishnan, Linear Matrix Inequalities in System and Control Theory, Vol. 15 of Studies in Applied Mathematics, SIAM, Philadelphia, 1994.

[61] K. H. Johansson, The quadruple-tank process: a multivariable laboratory process with an adjustable zero, IEEE Transactions on Control Systems Technology 8 (3) (2000) 456-465.

[62] S. Montes de Oca, V. Puig, Fault diagnosis of nonlinear systems using LPV interval observers, in: Proceedings of the 20th International Workshop on Principles of Diagnosis, 2009. 